\title{
miR-132 is upregulated in polycystic ovarian syndrome and inhibits granulosa cells viability by targeting Foxa1
}

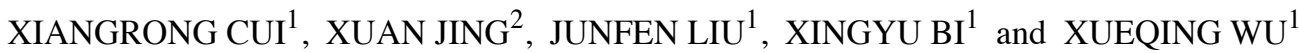 \\ ${ }^{1}$ Reproductive Medicine Center, Shanxi Women and Infants Hospital, Affiliate of Shanxi Medical University; \\ ${ }^{2}$ Department of Clinical Laboratory, Shanxi Provincial People's Hospital, Affiliate of Shanxi Medical University, \\ Taiyuan, Shanxi 030001, P.R. China
}

Received February 19, 2020; Accepted September 15, 2020

DOI: $10.3892 / \mathrm{mmr} .2020 .11590$

\begin{abstract}
Polycystic ovary syndrome (PCOS) is one of the most common endocrine metabolic disorders characterized by hyperandrogenism, polycystic ovaries and ovulatory dysfunction. Several studies have suggested that the aberrant expression of microRNAs (miRNAs/miRs) plays an important role in the pathogenesis of PCOS; however, the role and underlying mechanisms of miR-132 in the development of PCOS remain unclear. In the present study, the expression of miR-132 in granulosa cells (GCs) derived from 26 patients with PCOS and 30 healthy controls was detected by reverse transcription-quantitative PCR (RT-qPCR). The apoptosis of GCs was examined using a TUNEL assay. The human ovarian granulosa-like tumor cell line, KGN, was cultured for Cell Counting Kit-8 assays following the overexpression or knockdown of miR-132. TargetScan was applied to identify the potential targets of miR-132, which was further verified by a luciferase assay, RT-qPCR and western blotting. The expression of miR-132 was decreased in GCs from patients with PCOS. Moreover, the GCs of patients with PCOS exhibited significantly increased apoptotic nuclei. Furthermore, the overexpression of miR-132 inhibited the viability of KGN cells. In addition, the results verified that miR-132 directly targeted forkhead box protein A1 (Foxa1), the knockdown of which suppressed KGN cell viability. On the whole, the findings of the present study demonstrated that miR-132 inhibited cell viability and induced apoptosis by directly interacting with Foxa1. Thus, miR-132 may be a potential target for the treatment of patients with PCOS.
\end{abstract}

Correspondence to: Professor Xuan Jing, Department of Clinical Laboratory, Shanxi Provincial People's Hospital, Affiliate of Shanxi Medical University, 29 Shuang Ta East, Taiyuan, Shanxi 030001, P.R. China

E-mail: jx05070103@163.com

Key words: polycystic ovary syndrome, ovarian granulosa cells, microRNA-132, forkhead box protein A1, viability

\section{Introduction}

Polycystic ovary syndrome (PCOS) is one of the most prevalent endocrine metabolic disorders affecting $5-10 \%$ women of reproductive age globally $(1,2)$. It is generally characterized by hyperandrogenism, polycystic ovaries and anovulation; however, it is also associated with metabolic dysfunction, cardiovascular disease risk, abnormal granulosa cell (GC) proliferation and the arrest of follicle growth $(3,4)$. The clinical symptoms and signs of PCOS include an absent or irregular menstruation, difficulty in becoming pregnant, acne, and thick, dark and smooth skin patches (5). Genetic and environmental factors play a major role in the pathogenesis of PCOS; however, the underlying molecular mechanisms remain to be fully elucidated (6).

MicroRNAs (miRNAs or miRs) are small non-coding RNAs that negatively regulate protein-coding gene expression through RNA silencing and post-transcriptional regulation $(7,8)$. Studies have demonstrated that the aberrant expression of miRNAs is associated with the pathological progression of various diseases, including cancer, metabolic diseases, inflammation and reproductive disorders (8-10). However, the function and underlying molecular mechanisms of miRNAs in follicular development and in the development of PCOS have not yet been fully elucidated.

miR-132, located in the intron of a non-coding gene on chromosome 17 in humans, has been reported to play differential roles in various diseases, such as vascular endothelial inflammation, gestational diabetes mellitus and periodontitis (9-11). Recently, it was demonstrated that miR-132 can mediate tumor initiation and development by regulating cancer cell proliferation, apoptosis, invasion and migration $(12,13)$. Furthermore, Wu et al (14) revealed that miR-132 promoted estradiol synthesis in ovarian GCs via the translational repression of nuclear receptor subfamily 4 group A member 2 . However, the role and underlying mechanisms of miR-132 in PCOS remain unclear. Therefore, the aim of the present study was to determine whether miR-132 is involved in the abnormal viability of GCs from patients with PCOS and to elucidate the potential underlying mechanisms.

\section{Materials and methods}

Patients and samples. The study population consisted of women referred to the Reproductive Medicine Center of 
Shanxi Women and Infants Hospital (Taiyuan, China) between June 2016 and December 2016. All subjects were Han ethnic, from the Shanxi Province, in North China. The study was approved by the Ethics Committee of Shanxi Women and Infants Hospital Ethics (approval no. 201922021), and all the participants signed written informed consent for participation in this study. The blood and follicular fluid samples were obtained from 26 patients with PCOS and 30 healthy controls. Diagnosis of PCOS was based on the Rotterdam Criteria (15), including oligo-ovulation and/or anovulation, excess androgen activity, and ultrasound image of polycystic ovaries. Patients with endometriosis, congenital adrenal hyperplasia, hypothyroidism, androgen-secreting tumors, Cushing's syndrome and other systemic diseases were excluded from the study. The controls were patients with regular menstrual cycles who were infertile as a result of tubal factors (primarily tubal obstruction and abnormal tubal peristalsis) and/or male factors (including severe oligospermia and severe asthenospermia). All participants consented to endocrine tests and other routine checks, and the results are listed in Table I.

Controlled ovarian hyperstimulation protocol. Both patients with PCOS and control patients received in vitro fertilization and embryo transfer treatments, following standard operation procedure. All women underwent controlled ovarian hyper-stimulation with gonadotropin releasing hormone agonist long protocol commenced pituitary suppression with leuprolide acetate (Diphereline $0.1 \mathrm{mg}$; GenSci Company) at a dose of $0.05 \mathrm{mg} / \mathrm{d}$, during the mi-luteal phase of the preceding cycle. Complete pituitary suppression was confirmed by a serum follicle-stimulating hormone (FSH) level $<5 \mathrm{mIU} / \mathrm{ml}$, luteinizing hormone ( $\mathrm{LH}$ ) level $<5 \mathrm{mIU} / \mathrm{ml}$, estradiol $\left(\mathrm{E}_{2}\right)$ level $<50 \mathrm{pg} / \mathrm{ml}$, bilateral antral follicle diameter $<5 \mathrm{~mm}$, endometrial thickness $\leq 5 \mathrm{~mm}$. Urofollitropin (LIVZON) were used at doses ranging between $75 \mathrm{IU} /$ day and 300 IU/day in accordance with patient age, body mass index, size and number of antral follicles, and serum basic FSH level. The dosage of urofollitropin was adjusted according to ovarian response, which was assessed by ultrasound and serum $\mathrm{E}_{2}$ levels. Recombinant human choriogonadotropin-alfa solution (hCG; Merck Serono SpA) was administered subcutaneously at the $250 \mu \mathrm{g}$ dosing level when at least two follicles with $\geq 18 \mathrm{~mm}$ average diameter were detected. Oocyte retrieval was performed under the guidance of transvaginal ultrasounds 34 to $36 \mathrm{~h}$ after the hCG injection. Human GCs were obtained from follicular fluid at the same time.

Cell culture. After oocyte retrieval, all follicular fluids from each patient were pooled and stored in a tube. The GCs were prepared and cultured as described previously (16). Briefly, the aspirated follicular fluid was centrifuged at $1,000 \mathrm{x} g$ for $10 \mathrm{~min}$ at $37^{\circ} \mathrm{C}$ after removal of oocytes. The cell pellet was resuspended in $1 \mathrm{ml}$ phosphate buffer saline (PBS). Then, the suspension was overlayed on $1 \mathrm{ml}$ Ficoll, and centrifuged at $800 \mathrm{x}$ g for $30 \mathrm{~min}$ at $37^{\circ} \mathrm{C}$. GCs were aspirated from the interface and washed a few times with PBS. Next, the isolated and purified GCs $\left(1 \times 10^{5}\right)$ were cultured in Dulbecco's modified Eagle medium/nutrient mixture F12 Ham medium (DMEM/F12; Invitrogen; Thermo Fisher Scientific, Inc.) with $10 \%$ fetal bovine serum (FBS; TBD), $100 \mu \mathrm{g} / \mathrm{ml}$ penicillin and $0.1 \mathrm{mg} / \mathrm{ml}$ streptomycin (MRC) at $37^{\circ} \mathrm{C}$ with $5 \% \mathrm{CO}_{2}$ and $95 \%$ humidity.
Cell transfection. Human granulosa-like tumor cell line, KGN cells, which have the physiological characteristics of ovarian cells, were purchased from the American Type Culture Collection. GCs $\left(1 \times 10^{5}\right)$ were grown in DMEM/F12 with $10 \% \mathrm{FBS}$ at $37^{\circ} \mathrm{C}$ with $5 \% \mathrm{CO}_{2}$ and $95 \%$ humidity. The miR-132 mimics (cat. no. 219600), inhibitor (cat. no. 219300) and negative control (NC; cat. no. 1022076) were designed and synthesized by Qiagen, Inc. Forkhead box protein A1 (Foxa1)-small interfering (si)RNA (cat. no. A10001), control siRNA (si-NC; cat. no. A06001) were designed and synthesized by Shanghai GenePharma Co.,Ltd. Cells were seeded in 6-well plates $\left(2 \times 10^{5}\right.$ cells/well $) 1$ day before transfection to reach a confluency of $90 \%$, and then the medium was replaced with serum- and antibiotic-free medium. Then, miR-132 mimic, miR-132 inhibitor, and si-Foxal were transfected at a final concentration of $50 \mathrm{nM}$ using Lipofectamine ${ }^{\circledR} 2000$ reagent (Invitrogen; Thermo Fisher Scientific, Inc.) following the manufacturer's protocols. At $36 \mathrm{~h}$ after transfection, cells were collected for the following assays.

Cell viability assay. Cell viability was assessed using the Cell Counting Kit-8 (CCK-8) method. To explore the effect of miR-132 and Foxa1 on viability, cells transfected with miRNAs or siRNAs were plated in 96 -well plates at $5 \times 10^{3}$ cells/well. Cell viability was detected at 24,48 and $72 \mathrm{~h}$ after transfection using CCK-8 at $45 \mathrm{~nm}$ according to the manufacturer's instructions (Beyotime Institute of Biotechnology) at $37^{\circ} \mathrm{C}$ for $4 \mathrm{~h}$.

TUNEL assay. Apoptosis of GCs was determined by TUNEL staining using a TUNEL cell apoptosis detection kit (Nanjing KeyGen Biotech Co., Ltd.) according to the manufacturer's protocol. GCs $\left(1 \times 10^{4}\right)$ were cultured directly on coverslips. Subsequently, GCs were fixed in $4 \%$ paraformaldehyde at $4^{\circ} \mathrm{C}$ for $25 \mathrm{~min}$ and then washed thrice in PBS. The cells were incubated in $50 \mu \mathrm{l}$ permeabilisation solution $(0.2 \%$ Triton X-100) for $5 \mathrm{~min}$ at room temperature. GCs were then transferred into $50 \mu \mathrm{l}$ TUNEL reaction mixture ( $45 \mu \mathrm{l}$ equilibrium buffer $+5 \mu \mathrm{l}$ nucleotide mixture $+1 \mu 1 \mathrm{TdT}$ enzyme) and incubated in a humidified chamber for $60 \mathrm{~min}$ in the dark at $37^{\circ} \mathrm{C}$. The reaction was terminated by incubating the GCs in $2 \mathrm{X}$ SSC buffer for $15 \mathrm{~min}$ at room temperature. The cells were then washed three times with PBS/PVP. Subsequently, cells were treated with $0.5 \mu \mathrm{g} / \mathrm{ml}$ of DNase-free RNase. Images were captured using a BX40 microscope (BX40; Olympus Corporation). The buffy (faint yellow) nucleus indicated TUNEL-positive cells. A total of five fields (magnification, $\mathrm{x} 200$ ) were taken randomly for each sample. Data are reported as the percentage of TUNEL-positive cells among the total number of cells. Each experiment was performed in triplicate. Negative control cells were subjected to the TUNEL assay without the addition of terminal deoxynucleotidyl transferase in the reaction mixture. Positive control cells were incubated with $100 \mu \mathrm{l}$ DNase I solution prior to the TUNEL assay to induce DNA strand degradation.

$R N A$ isolation and reverse transcription-quantitative PCR $(R T-q P C R)$ assay. Total RNA of GCs was extracted using the RNeasy/miRNeasy Mini kit (Qiagen Benelux BV), according to the manufacturer's instructions. Total RNA (2 ng) was used for reverse transcription using the OneStep RT-PCT kit (Qiagen Benelux BV), following the manufacturer's instructions. 
Table I. Characteristics of patients with PCOS $(n=26)$ and the controls $(n=30)$.

\begin{tabular}{lccc}
\hline Characteristics & PCOS group & Control group & P-value \\
\hline Age, years & $29.86 \pm 2.81$ & $30.11 \pm 2.95$ & 0.75 \\
BMI & $23.74 \pm 3.36$ & $21.84 \pm 3.02$ & 0.03 \\
Infertility duration, & $4.42 \pm 2.76$ & $4.78 \pm 2.99$ & 0.64 \\
years & & & \\
FSH, mIU/ml & $6.79 \pm 2.18$ & $6.96 \pm 1.92$ & 0.76 \\
LH, mIU/ml & $9.53 \pm 6.48$ & $4.37 \pm 2.11$ & $<0.001$ \\
E $_{2}, \mathrm{pg} / \mathrm{ml}$ & $51.12 \pm 14.77$ & $47.96 \pm 19.98$ & 0.51 \\
PRL, ng/ml & $14.13 \pm 6.48$ & $14.41 \pm 6.26$ & 0.87 \\
TES, ng/dl & $1.14 \pm 0.39$ & $0.44 \pm 0.17$ & $<0.001$ \\
\end{tabular}

All data are expressed as the mean \pm SD. BMI, body mass index; FSH, follicle-stimulating hormone; $\mathrm{LH}$, luteinizing hormone; $\mathrm{E}_{2}$, estradiol; TES, testosterone; PRL, prolactin; PCOS, polycystic ovary syndrome.

The primers for miR-132 were the exact sequence of mature miR-132. U6 was used as the internal control. The primers were purchased from Qiagen Benelux BV. The thermocycling conditions for miRNA were as follows: $95^{\circ} \mathrm{C}$ for $15 \mathrm{~min}$, followed by $95^{\circ} \mathrm{C}$ for $15 \mathrm{sec}$, and $60^{\circ} \mathrm{C}$ for $1 \mathrm{~min}$ (40 cycles). For the mRNA expression of Foxa1, GAPDH was used as the internal control. The primer sequences for Foxal and GAPDH were as follows: Foxa1 sense, 5'-AGGGCTGGATGGTTGTATTG-3' and antisense, 5'-GCCTGAGTTCATGTTGCTGA-3'; GAPDH sense, 5'-GAAGGTGAAGGTCGGAGTC-3' and antisense, 5'-GAA GATGGTGATGGGATTTC-3'. The thermocycling conditions for Foxal were as follows: $95^{\circ} \mathrm{C}$ for $5 \mathrm{~min} ; 35$ cycles of $95^{\circ} \mathrm{C}$ for $1 \mathrm{~min}$, and $60^{\circ} \mathrm{C}$ for $1 \mathrm{~min}$ and $72^{\circ} \mathrm{C}$ for $1 \mathrm{~min}$; and then $72^{\circ} \mathrm{C}$ for $7 \mathrm{~min}$. RT-qPCR was performed in triplicate using a SYBR ${ }^{\circledR}$ Premix Ex Taq Kit (Takara Bio, Inc.), according to the manufacturer's protocol, on a CFX96 Real-time PCR system (Bio-Rad Laboratories, Inc.). All reactions were run in triplicate and gene expression was determined using the $2^{-\Delta \Delta \mathrm{Cq}}$ method (17).

Dual-luciferase reporter assay. Foxa1 was predicted to be a target of miR-132 by the online database TargetScanHuman 7.1 (www.targetscan.org; Whitehead Institute for Biomedical Research). Then, the fragment was inserted into the pGL3 luciferase promoter vector (Promega Corporation) to develop the Luc-pGL3-Foxa1-3'UTR and Luc-pGL3-Foxa1-mut-3'UTR vectors. Cells $\left(1 \times 10^{5}\right)$ in 24-well plates were co-transfected with Luc-pGL3-Foxa1-3'UTR orLuc-pGL3-Foxa1-mut-3'UTR vector and miR-132 mimics or miR-NC using Lipofectamine 2000 reagent, according to the instructions of the manufacturer. The Renilla luciferase reporter vector was transfected as an internal control in each assay. At $48 \mathrm{~h}$ post-transfection, firefly and Renilla luciferase activities were detected using a dual-luciferase reporter system (Promega Corporation). The results are expressed as relative luciferase activity (Firefly/Renilla). All experiments were performed three times in triplicate.

Western blot analysis. Western blotting was performed to evaluate the expression of Foxa1. Briefly, the cells were collected and lysed on ice in RIPA lysis buffer (Beyotime

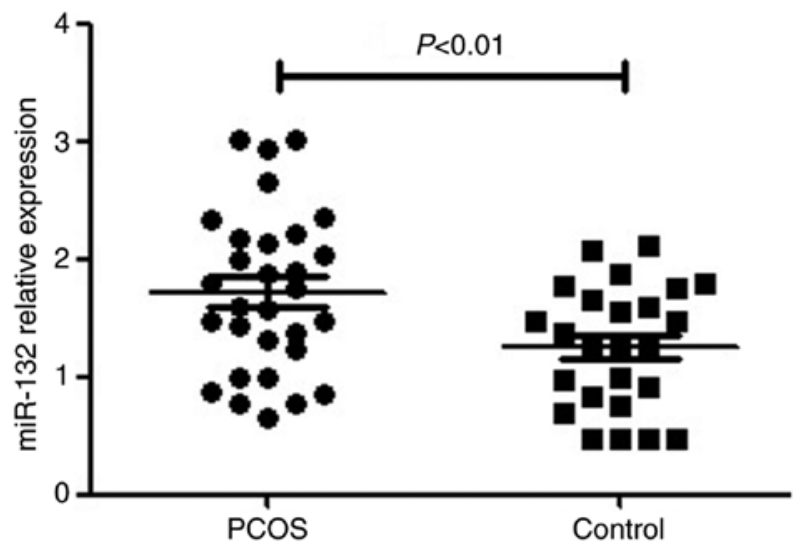

Figure 1. miR-132 expression is upregulated in human GCs from patients with PCOS. Expression levels of miR-132 were examined by reverse transcription-quantitative PCR in isolated human GCs from the aspirated follicular fluid of patients with PCOS ( 26 cases) and controls ( 30 cases). $\mathrm{P}<0.01$ vs. controls. GC, granulosa cell; PCOS, polycystic ovary syndrome; miR, microRNA.

Institute of Biotechnology) with protease inhibitor, according to the manufacturer's instructions. The protein concentration of cell lysates was determined using a BCA kit (Wuhan Boster Biological Technology Co., Ltd.). Equal amounts of protein lysates (30 $\mu \mathrm{g}$ per lane) were resolved by $10 \%$ SDS-PAGE, and then electrotransferred to PVDF membranes (EMD Millipore). The membranes were blocked with TBS with $0.1 \%$ Tween-20 (TBST)_containing 5\% non-fat milk for $2 \mathrm{~h}$ at room temperature, and then incubated with the specific antibodies at $4^{\circ} \mathrm{C}$ overnight, including mouse anti-Foxa1 (1:1,000; cat. no. ab40868), mouse anti-Bax (1:1,000; cat. no. ab3191), mouse anti-Bcl-2 (1:1,000; cat. no. ab692) and mouse anti-GAPDH (1:200; cat. no. ab8245) monoclonal antibodies (both from Abcam). After washing with TBST, the membranes were further incubated with HRP-conjugated goat anti-mouse IgG (1:2,000; cat. no. BA1051; Wuhan Boster Biological Technology Co., Ltd.) at $37^{\circ} \mathrm{C}$ for $1 \mathrm{~h}$, followed by visualization with an ECL kit (Nanjing KeyGen Biotech Co., Ltd.). Protein expression levels were semi-quantified using Quantity One software (version 4.6.7; Bio-Rad Laboratories, Inc.).

Statistical analysis. All statistical analyses were performed using GraphPad Prism 6.0 (GraphPad software, Inc.). Normally distributed data are presented as the mean \pm standard error of the mean. To check the normality of the distribution, the Shapiro-Wilk test was performed. Two-tailed Student's t-test was performed for comparisons of the mean values of two groups; one-way ANOVA (followed by a Bonferroni post hoc test) was used to determine differences among the mean values of multiple groups because the quantitative data followed a normal distribution. $\mathrm{P}<0.05$ was considered to indicate a statistically significant difference.

\section{Results}

Patient characteristics. The characteristics of female patients in the PCOS group and control group are summarized in Table I. In total, 26 patients with PCOS were recruited from patients seeking reproductive assistance at the Reproductive Medicine Center of Shanxi Women and Infants Hospital. 
A Positive control

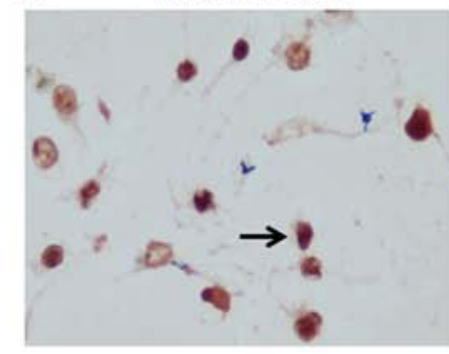

Negative control

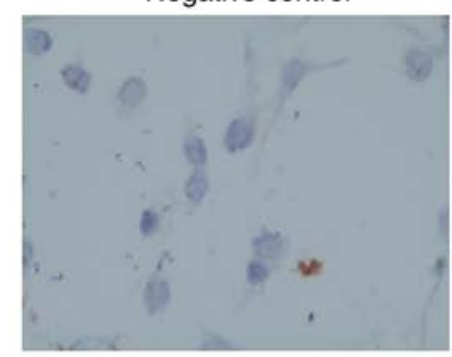

PCOS group

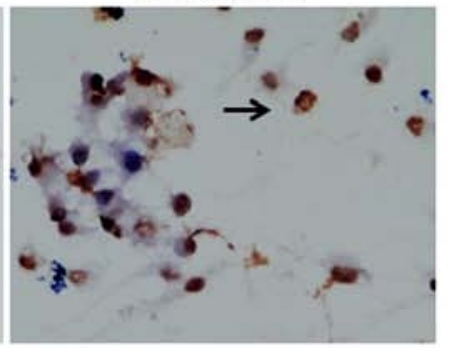

Control group

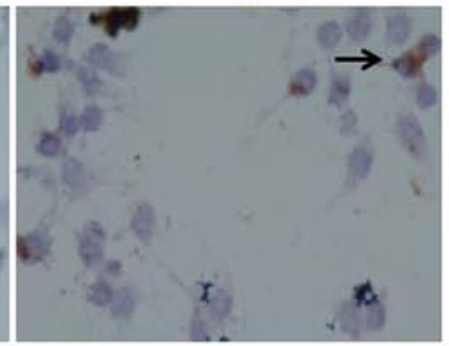

$\mathrm{B}$

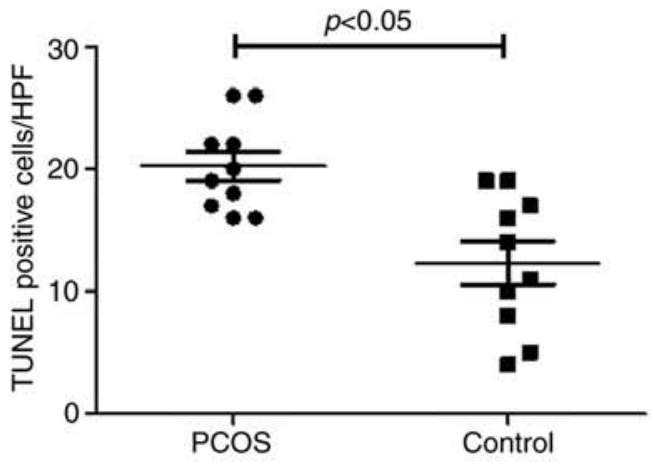

Figure 2. TUNEL assay showing the apoptosis of granulosa cells. (A) The buffy (faint yellow) nucleus indicated TUNEL-positive cells. Slides were observed under a microscope (magnification, x200). (B) Statistical analysis of TUNEL results. PCOS, polycystic ovary syndrome.

Each patient with PCOS was confirmed clinically. A total of 16 cases of tubal infertility and 14 cases of infertility related to male factors were included as controls for this study. There was no significant difference between the PCOS and control groups regarding age, infertility duration, and levels of $\mathrm{FSH}$, $\mathrm{E}_{2}$ and PRL. However, compared with the control group, the $\mathrm{BMI}$, and levels of $\mathrm{LH}$ and TES were significantly increased in the PCOS group $(\mathrm{P}<0.05)$

miR-132 expression is upregulated in human GCs from patients with PCOS. RT-qPCR was performed to detect miR-132 expression in GCs of 26 patients with PCOS and 30 controls. Compared with the controls, the expression of miR-132 was significantly upregulated in the GCs of patients with PCOS ( $\mathrm{P}<0.05$; Fig. 1).

Apoptosis is increased in GCs from patients with PCOS. In order to determine the GC apoptotic rate in PCOS, cells were cultured directly on coverslips and stained with a TUNEL cell apoptosis detection kit. A significantly increased number of apoptotic nuclei were present in the PCOS group compared with the control group $(\mathrm{P}<0.05$; Fig. $2 \mathrm{~A}$ and $\mathrm{B})$.

miR-132 negatively regulates cell growth and viability in GCs. Having noted a significantly higher expression of miR-132 in GCs of the ovaries from patients with PCOS, it was proposed that miR-132 may be associated with the growth and viability of GCs. Therefore, GCs were transfected with miR-132 mimics and miR-132 inhibitor, and the relative miR-132 expression was verified (Fig. 3A and B). As shown in Fig. 3C, the CCK-8 assay revealed that decreased expression of miR-132 in GCs promoted viability $(\mathrm{P}<0.05)$. In contrast to the miR-132 mimics, the miR-132 inhibitor promoted cell growth $(\mathrm{P}<0.05$; Fig. 3C). To further determine the role of miR-132 in cell apoptosis of human GCs, western blotting was used to detect the protein expression levels of Bax and
Bcl-2. The results showed that miR-132 mimics significantly increased Bax protein expression and decreased protein expression of Bcl-2 when compared with the control group. Whereas, the miR-132 inhibitor significantly upregulated the protein expression of $\mathrm{Bcl}-2$ and decreased the protein expression of Bax when compared with the control group $(\mathrm{P}<0.05$; Fig. 3D and $\mathrm{E})$.

miR-132 directly inhibits Foxal expression by binding to its 3'UTR. Foxal was predicted to be a target of miR-132 by the online database TargetScanHuman 7.1 (www.targetscan.org), with the sequence GACUGUUA in its 3'UTR being the predicted binding site (Fig. 4A). A significant downregulation of Foxal expression was detected by RT-qPCR in human GCs from patients with PCOS ( $\mathrm{P}<0.05$; Fig. 4B). Then, RT-qPCR and western blotting were performed to observe the expression of Foxa1 at the mRNA and protein levels in GCs transfected with miR-132 mimics and inhibitor. Compared with the control group, following the upregulation of miR-132, the mRNA and protein levels of Foxal were significantly decreased. Whereas, following the downregulation of miR-132, Foxa1 mRNA and protein levels were significantly increased $(\mathrm{P}<0.05$; Fig. 4C, D and F). To further demonstrate whether Foxal was a direct target of miR-132, Foxa1 3'UTR was cloned into a luciferase reporter vector and the putative miR-132 binding site in the Foxal 3'UTR was mutated (Fig. 4E). Compared with the control group, miR-132 overexpression suppressed the luciferase activity of the reporter vector with Foxa1-WT; whereas miR-132 knockdown increased the luciferase activity of the WT vector. Moreover, the luciferase activity of the MUT vector in GCs was not affected by miR-132 overexpression or knockdown (Fig. 4E). Taken together, these data suggested that the Foxal gene is a direct target of miR-132 overexpression, which inhibits Foxa1 expression in GCs. 

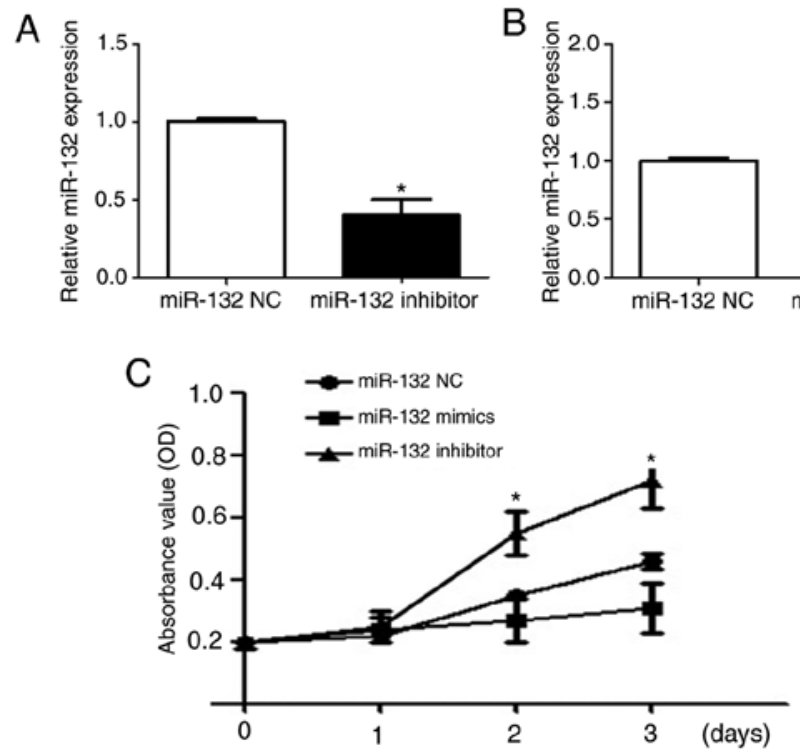

$\mathrm{B}$

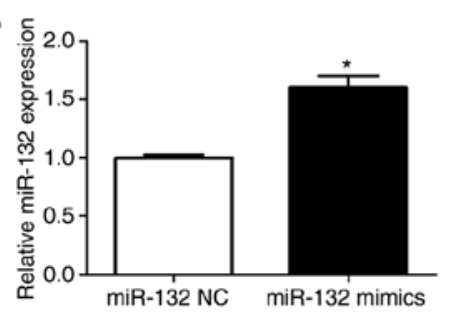

E
D

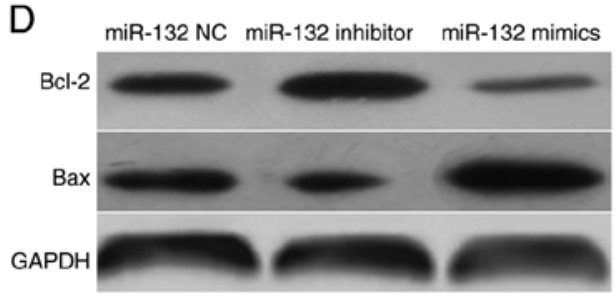

a miR-132 NC

a miR-132 inhibitor

a miR-132 mimics

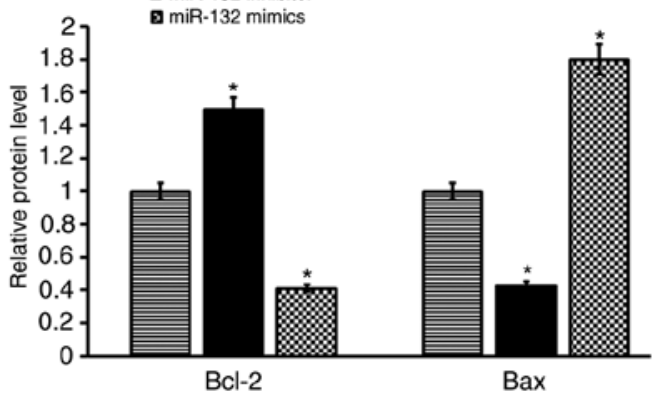

Figure 3. miR-132 negatively regulates cell growth and viability of GCs. (A and B) Relative miR-132 expression following transfection with mimics and inhibitor. (C) Cell viability was determined by a Cell Counting Kit-8 assay in KGN cells transfected with miR-132 NC, miR-132 inhibitor or miR-132 mimics. (D) Protein expression levels of Bax and Bcl-2 in GCs were examined by western blotting with GAPDH as an internal reference. (E) Semi-quantification of the western blotting results. "P<0.05 vs. miR-132 NC. miR, microRNA; GC, granulosa cell; NC, negative control.

A

Foxa1 3' UTR 5'... UCCCCCAGUGCAAAAGACUGUUA.... 3' IIIIII hsa-miR-132-3p $\quad 3^{\prime} \ldots$ GCUGGUACCGACAUCUGACAAU. ... 5' mut-Foxa1 3' UTR 5'... UCCCCCAGUGCAAAAGUGAGAAA... .3'

C

\begin{tabular}{lcc} 
Control & $\begin{array}{c}\text { miR-132 } \\
\text { mimics }\end{array}$ & $\begin{array}{c}\text { miR-132 } \\
\text { inhibitor }\end{array}$ \\
Foxa1 & \\
\hline
\end{tabular}

E

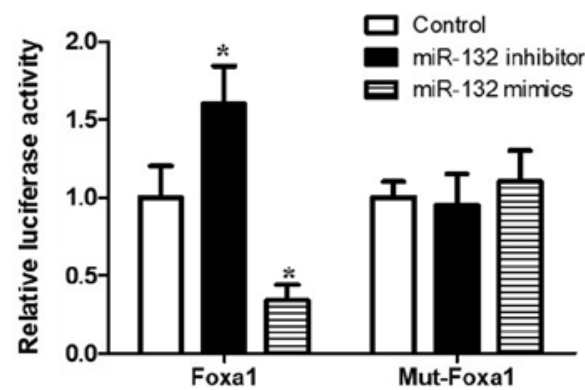

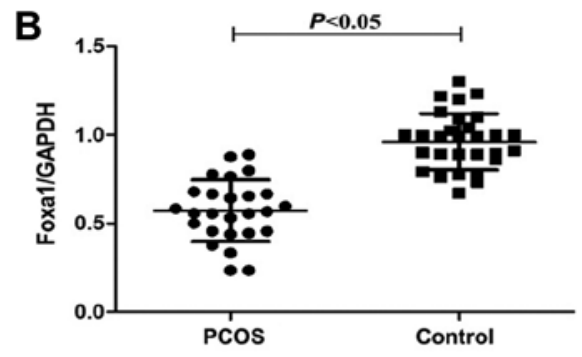
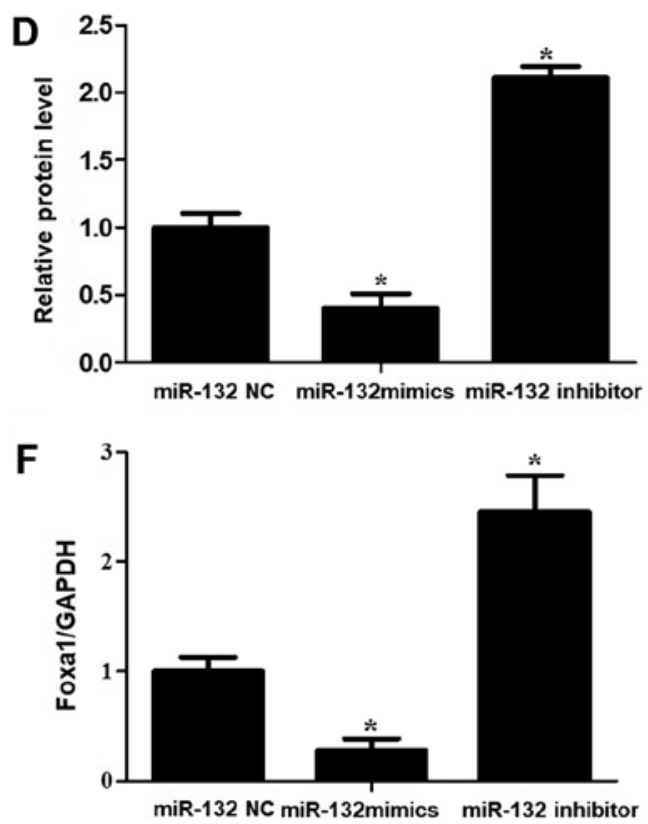

Figure 4. miR-132 directly inhibits Foxa1 expression by binding to its 3'UTR. (A) The sequence GACUGUUA in Foxa1 3'UTR was predicted to be the binding site of miR-132. Five bases in the binding site were mutated to construct mut-Foxal 3'UTR. (B) The mRNA expression of Foxal was examined by reverse transcription-quantitative PCR in isolated human granulosa cells from the aspirated follicular fluid of patients with PCOS and controls. (C) Western blotting data showed the protein expression of Foxal following transfection with mimics and inhibitors. (D) Semi-quantification of the western blotting results. (E) Validation of miR-132 binding to the Foxa1 3'UTR using a dual-luciferase reporter assay. (F) Foxal mRNA expression was inhibited by miR-132 overexpression and upregulated by transfection with a miR-132 inhibitor. GAPDH was used as an internal reference. "P $<0.05$ vs. miR-132 NC. miR, microRNA; NC, negative control; Foxa1, forkhead box protein A1; UTR, untranslated region; PCOS, polycystic ovary syndrome; mut, mutant. 

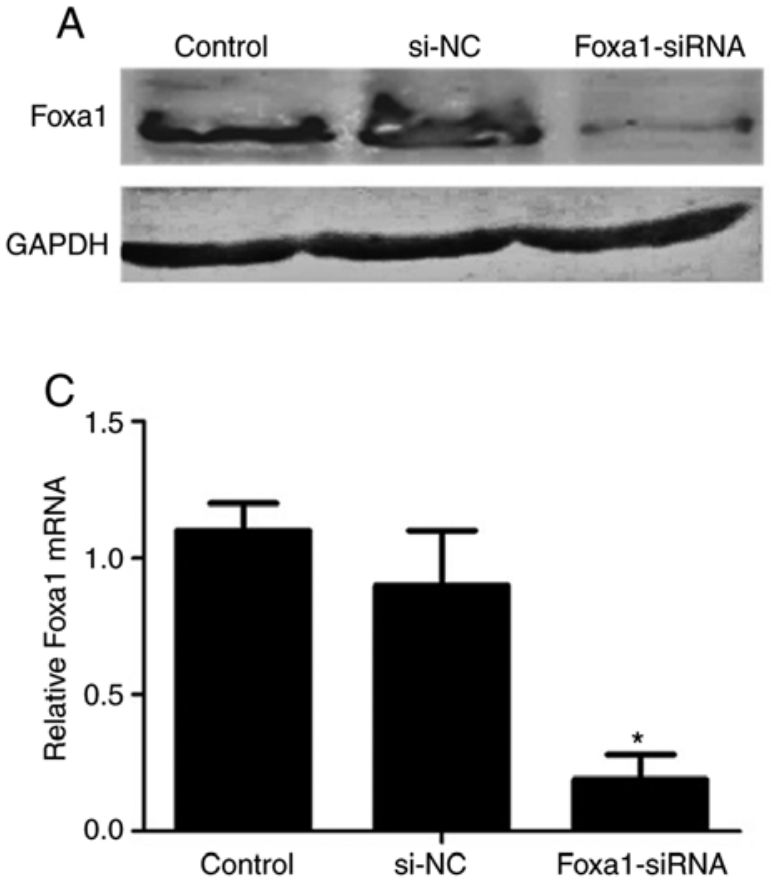
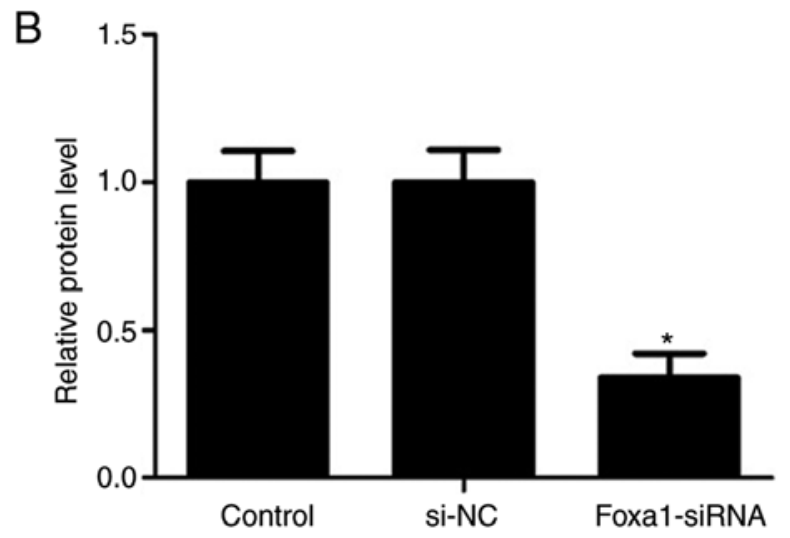

D

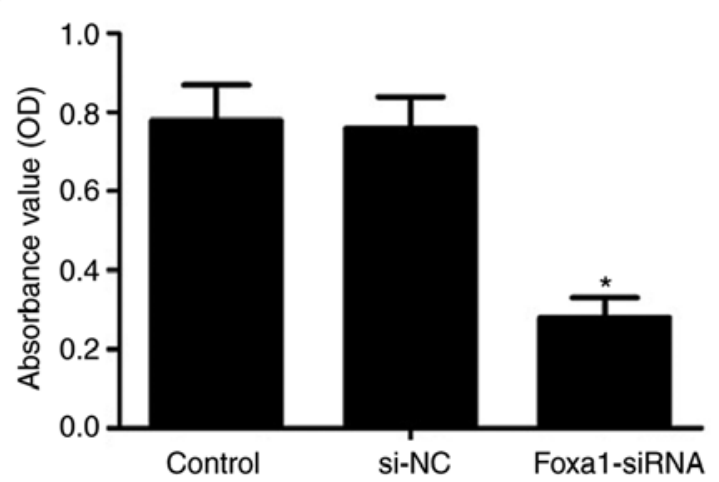

Figure 5. Silencing of Foxal inhibits the viability of human GCs. (A) Western blotting data showed the protein expression of Foxa1 in GCs transfected with si-NC or Foxa1-siRNA. (B) Semi-quantification of the western blotting results. (C) Reverse transcription-quantitative PCR analysis showed the mRNA expression of Foxa1 in GCs transfected with si-NC or Foxa1-siRNA. (D) A Cell Counting Kit-8 assay was performed to evaluate the viability of GCs transfected with Foxa1-siRNA. " $\mathrm{P}<0.05$ vs. si-NC. siRNA, small interfering RNA; Foxa1, forkhead box protein A1; GC, granulosa cell; NC, negative control.

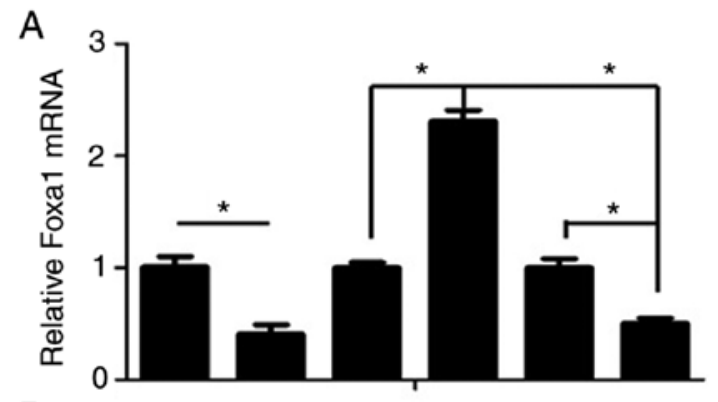

B

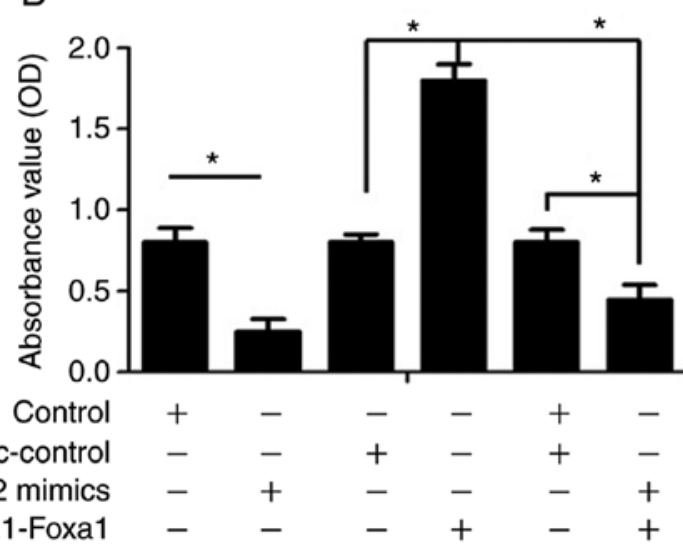

Figure 6. Foxa1 overexpression reverses the inhibitory effect of miR-132 mimics. (A) Foxa1 mRNA expression in human GCs transfected with miR-132 mimics or pcDNA3.1-Foxa1 was determined by reverse transcriptionquantitative PCR. (B) A Cell Counting Kit- 8 assay was performed to evaluate the viability of GCs. ${ }^{*} \mathrm{P}<0.05$. Foxa1, forkhead box protein $\mathrm{A} 1$; miR, microRNA; GC, granulosa cell.
Cytotoxicity assay of silencing Foxalin human GCs. To explore the function of Foxal in GCs, Foxal expression was knocked down by siRNA in human GCs from control patients. RT-qPCR and western blotting indicted that mRNA and protein levels of Foxal were significantly decreased after $24 \mathrm{~h}$ in GCs transfected with Foxal-siRNA ( $\mathrm{P}<0.05$; Fig. 5A-C). Then, cell viability detection was performed to evaluate the effect of Foxal-siRNA on human GCs. As expected, Foxa1 siRNA-transfected GCs displayed reduced cell viability compared with si-NC at $48 \mathrm{~h}$ post-transfection $(\mathrm{P}<0.05$; Fig. 5D). These results indicated that silencing Foxa1 inhibited human GC growth.

Foxal overexpression reverses the suppressive effect of miR-132 mimics. To further determine the role of miR-132 on cell viability through the direct targeting of Foxa1 in human GCs, cells were transfected with pcDNA3.1-Foxa1 or miR-132 mimics. As shown in Fig. 6A, overexpression of Foxa1 by pcDNA3.1-Foxa1 significantly increased Foxa1 expression in human GCs compared with the vector control group $(\mathrm{P}<0.05)$ Furthermore, the inhibitory effect of miR-132 mimics on Foxa1 expression was partially reversed by Foxal overexpression. Subsequently, it was found that the suppressed cell viability following transfection with miR-132 mimics was attenuated by Foxal overexpression in human GCs ( $\mathrm{P}<0.05$; Fig. 6B).

\section{Discussion}

The aim of the present study was to examine whether miR-132 was involved in the abnormal viability of GCs from patients 
with PCOS and to elucidate the underlying mechanisms. It was observed that the expression of miR-132 was significantly increased in GCs from patients with PCOS. Furthermore, the results revealed that the decreased expression of miR-132 was associated with a increased cell apoptotic index of GCs from patients with PCOS. Moreover, the dual-luciferase reporter assay revealed that Foxal was a direct target for miR-132 and promoted GC viability. Foxal overexpression reversed the suppressive effects of miR-132 mimics. These results thus indicated that miR-132 suppressed cell viability, and the potential underlying mechanisms are associated with the targeting and suppression of Foxal expression.

Previous studies have demonstrated that miR-132 plays a pro-apoptotic role in a number of cancer types, such as glioma (18), colorectal cancer $(19,20)$, osteosarcoma (21), hepatic carcinoma (22), breast cancer (23), pituitary tumor (24) and lung cancer (25). In addition, an increased expression of miR-132 has been reported in periovulatory mouse GCs following $\mathrm{LH} / \mathrm{hCG}$ treatment and enhanced estradiol synthesis in GCs $(14,26)$. However, a large number of studies on miR-132 have primarily focused on cancer, and the mechanisms underlying the role of miR-132 in PCOS have not yet been investigated. To the best of our knowledge, the present study was the first to report that miR-132 was involved in inhibiting the viability of KGN cells, suggesting that miR-132 plays crucial roles in the abnormal viability of GCs, which may lead to the development of PCOS.

Foxa1 is a transcription factor that belongs to the forkhead family, consisting of the winged-helix DNA-binding domain, and the $\mathrm{N}$-terminal and $\mathrm{C}$-terminal transcriptional domains, thereby delineating genomic regions and allowing for the subsequent binding of other transcription factors, such as the estrogen receptor, progesterone receptor and androgen receptor (27-29). Foxa1 is expressed in a variety of organs, including breast, liver, pancreas and prostate, and can influence the expression of a large number of genes associated with metabolic processes, the regulation of signaling and the cell cycle $(30,31)$. It has been reported that Foxal is a direct target of miR-132 in breast cancer, thyroid cancer and nasopharyngeal carcinoma (32-34). Consistent with the aforementioned results, the present study further demonstrated that Foxa1 was a direct target of miR-132 in KGN cell viability. However, Sang et al (35), who identified miRNAs in the human follicular fluid of patients with PCOS, demonstrated that miR-132 was expressed at significantly lower levels in the follicular fluid of patients with PCOS compared with in the healthy controls. The differences were likely due to a variety of reasons. miR-132 expression in the aforementioned study was measured in human follicular fluid, whereas in the present study, miR-132 expression was examined in human GCs. In addition, the regulatory process of GC proliferation is complex and is closely related to a variety of factors $(36,37)$.

In conclusion, the findings of the present study demonstrated that the expression of miR-132 was significantly increased in patients with PCOS. In addition, the overexpression of miR-132 inhibited the viability of KGN cells by targeting Foxa1. These results provided novel evidence for the dysregulated viability of GCs observed in PCOS. Due to the limitation in the number of PCOS samples and cell types used, further investigations are required in order to fully determine the underlying molecular mechanisms of miR-132 in POCS.

\section{Acknowledgements}

Not applicable.

\section{Funding}

This study was supported by the Scientific Research Project of Shanxi Provincial Department of Health (grant no. 201601070), the Initial Scientific Research Fund of PhD at Shanxi Provincial People's Hospital (grant no. b201635), the Natural Science Foundation of Shanxi (grant nos. 201901D211519 and 201901D211546), the Natural Science Foundation of Shanxi (grant no. 201901D211546), the Research Project Supported by Shanxi Scholarship Council of China (grant no. HGKY2019092) and China Postdoctoral Science Foundation (grant no. 2020M670703).

\section{Availability of data and materials}

The datasets used and/or analyzed during the current study are available from the corresponding author on reasonable request.

\section{Authors' contributions}

$\mathrm{XC}$ and $\mathrm{XJ}$ wrote the manuscript and made substantial contributions to the design of the present study. XW, JL and XB contributed to data interpretation and writing of the manuscript. All authors read and approved the final manuscript.

\section{Ethics approval and consent to participate}

The present study was approved by the Ethics Committee of Shanxi Women and Infants Hospital Ethics (Taiyuan, China; approval no. 201922021), and all the participants signed written informed consent for participation in this study.

\section{Patient consent for publication}

Not applicable.

\section{Competing interests}

The authors declare that they have no competing interests.

\section{References}

1. Lin J, Huang J, Wang N, Kuang Y and Cai R: Effects of pre-pregnancy body mass index on pregnancy and perinatal outcomes in women with PCOS undergoing frozen embryo transfer. BMC Pregnancy Childbirth 19: 487, 2019.

2. Li S, Qi J, Tao Y, Zhu Q, Huang R, Liao Y, Yue J, Liu W, Zhao H, Yin $\mathrm{H}$ and Sun Y: Elevated levels of arachidonic acid metabolites in follicular fluid of PCOS patients. Reproduction: Nov 1, 2019 (Epub ahead of print). doi: 10.1530/REP-19-0136.

3. Sagvekar P, Mangoli V, Desai S, Patil A and Mukherjee S: LINE1 CpG-DNA hypomethylation in granulosa cells and blood leukocytes is associated with PCOS and related traits. J Clin Endocrinol Metab 102: 1396-1405, 2017. 
4. Makrinou E, Drong AW, Christopoulos G, Lerner A Chapa-Chorda I, Karaderi T, Lavery S, Hardy K, Lindgren CM and Franks S: Genome-wide methylation profiling in granulosa lutein cells of women with polycystic ovary syndrome (PCOS) Mol Cell Endocrinol 500: 110611, 2019.

5. Qiu X, Wei Y,Liu C,Ding C and Zhao S: Hyperandrogen enhances apoptosis of human ovarian granulosa cells via up-regulation and demethylation of PDCD4. Gynecol Endocrinol 36: 333-337, 2019.

6. Li Y, Liu YD, Zhou XY, Chen SL, Chen X, Zhe J, Zhang J, Zhang QY and Chen YX: MiR-29a regulates the proliferation, aromatase expression, and estradiol biosynthesis of human granulosa cells in polycystic ovary syndrome. Mol Cell Endocrinol 498: 110540, 2019.

7. Song Y, Yu G, Xiang Y, Li Y, Wan L and Tan L: Altered miR-186 and miR-135a contribute to granulosa cell dysfunction by targeting ESR2: A possible role in polycystic ovary syndrome Mol Cell Endocrinol 494: 110478, 2019.

8. Chen B, Xu P, Wang J and Zhang C: The role of MiRNA in polycystic ovary syndrome (PCOS). Gene 706: 91-96, 2019.

9. Wang W, Li X, Ren L, Yuan C, Han Y and Wang Z: MiR-132 relieves vascular endothelial inflammation and improve endothelial function in atherosclerosis rats by regulating SIRT1. Minerva Endocrinol 45: 158-161, 2019.

10. Han Y, Wang F, Shao L, Huang P and Xu Y: LncRNA TUG1 mediates lipopolysaccharide-induced proliferative inhibition and apoptosis of human periodontal ligament cells by sponging miR-132. Acta Biochim Biophys Sin (Shanghai) 51: 1208-1215, 2019.

11. Zhou $X$, Xiang $C$ and Zheng $X$ : miR-132 serves as a diagnostic biomarker in gestational diabetes mellitus and its regulatory effect on trophoblast cell viability. Diagn Pathol 14: 119, 2019.

12. Wei XC and Lv ZH: MicroRNA-132 inhibits migration, invasion and epithelial-mesenchymal transition via TGF $\beta 1 / \mathrm{Smad} 2$ signaling pathway in human bladder cancer. Onco Targets Ther 12: 5937-5945, 2019.

13. Zhang XL, Sun BL, Tian SX, Li L, Zhao YC and Shi PP: MicroRNA-132 reverses cisplatin resistance and metastasis in ovarian cancer by the targeted regulation on Bmi-1. Eur Rev Med Pharmacol Sci 23: 3635-3644, 2019.

14. Wu S, Sun H, Zhang Q, Jiang Y, Fang T, Cui I, Yan G and $\mathrm{Hu}$ Y: MicroRNA-132 promotes estradiol synthesis in ovarian granulosa cells via translational repression of Nurr1. Reprod Biol Endocrinol 13: 94, 2015.

15. Azziz R: Controversy in clinical endocrinology: Diagnosis of polycystic ovarian syndrome: The Rotterdam criteria are premature. J Clin Endocrinol Metab 91: 781-785, 2006.

16. Kaur S, Archer KJ, Devi MG, Kriplani A, Strauss JF III and Singh R: Differential gene expression in granulosa cells from polycystic ovary syndrome patients with and without insulin resistance: Identification of susceptibility gene sets through network analysis. J Clin Endocrinol Metab 97: E2016-E2021, 2012.

17. Livak KJ and Schmittgen TD: Analysis of relative gene expression data using real-time quantitative PCR and the 2(-Delta Delta C(T)) method. Methods 25: 402-408, 2001

18. Li Y, Zhang J, He J, Zhou W, Xiang G and Xu R: MicroRNA-132 cause apoptosis of glioma cells through blockade of the SREBP-1c metabolic pathway related to SIRT1. Biomed Pharmacother 78: $177-184,2016$

19. Mokutani Y, Uemura M, Munakata K, Okuzaki D, Haraguchi N Takahashi H, Nishimura J, Hata T, Murata K, Takemasa I, et al down-regulation of microrna-132 is associated with poor prognosis of colorectal cancer. Ann Surg Oncol 23 (Suppl 5): 599-608, 2016

20. Qin J, Ke J, Xu J, Wang F, Zhou Y, Jiang Y and Wang Z: Downregulation of microRNA-132 by DNA hypermethylation is associated with cell invasion in colorectal cancer. Onco Targets Ther 8: 3639-3648, 2015.
21. Liu Y, Li Y, Liu J, Wu Y and Zhu Q: MicroRNA-132 inhibits cell growth and metastasis in osteosarcoma cell lines possibly by targeting Sox4. Int J Oncol 47: 1672-1684, 2015.

22. Lei CJ, Li L, Gao X, Zhang J, Pan QY, Long HC, Chen CZ, Ren DF and Zheng G: Hsa-miR-132 inhibits proliferation of hepatic carcinoma cells by targeting YAP. Cell Biochem Funct 33: 326-333, 2015.

23. Zhang ZG, Chen WX, Wu YH, Liang HF and Zhang BX: MiR-132 prohibits proliferation, invasion, migration, and metastasis in breast cancer by targeting HN1. Biochem Biophys Res Commun 454: 109-114, 2014.

24. Renjie W and Haiqian L: MiR-132, miR-15a and miR-16 synergistically inhibit pituitary tumor cell proliferation, invasion and migration by targeting Sox 5. Cancer Lett 356: 568-578, 2015.

25. Li Y, Zu L, Wang Y, Wang M, Chen P and Zhou Q: miR-132 inhibits lung cancer cell migration and invasion by targeting SOX4. J Thorac Dis 7: 1563-1569, 2015.

26. Fiedler SD, Carletti MZ, Hong X and Christenson LK: Hormonal regulation of MicroRNA expression in periovulatory mouse mural granulosa cells. Biol Reprod 79: 1030-1037, 2008

27. Fu X, Pereira R, De Angelis C, Veeraraghavan J, Nanda S, Qin L, Cataldo ML, Sethunath V, Mehravaran S, Gutierrez C, et al: FOXA1 upregulation promotes enhancer and transcriptional reprogramming in endocrine-resistant breast cancer. Proc Natl Acad Sci USA 116: 26823-26834, 2019.

28. Jing X, Liang H, Hao C, Hongxia L and Cui X: Analyses of an epigenetic switch involved in the activation of pioneer factor FOXA1 leading to the prognostic value of estrogen receptor and FOXA1 co-expression in breast cancer. Aging 11: 7442-7456, 2019.

29. Gou L, Zou H and Li B: Long noncoding RNA MALAT1 knockdown inhibits progression of anaplastic thyroid carcinoma by regulating miR-200a-3p/FOXA1. Cancer Biol Ther 20: $1355-1365,2019$.

30. Stone L: Different FOXA1 classes drive prostate cancer. Nat Rev Urol 16: 508, 2019

31. BenAyed-Guerfali D, Dabbeche-Bouricha E, Ayadi W, Trifa F, Charfi S, Khabir A, Sellami-Boudawara T and Mokdad-Gargouri R: Association of FOXA1 and EMT markers (Twist1 and E-cadherin) in breast cancer. Mol Biol Rep 46: 3247-3255, 2019.

32. Wang D, Ren J, Ren H, Fu JL and Yu D: MicroRNA-132 suppresses cell proliferation in human breast cancer by directly targeting FOXA1. Acta Pharmacol Sin 39: 124-131, 2018.

33. Chen X, Li M, Zhou H and Zhang L: miR-132 targets FOXA1 and exerts tumor-suppressing functions in thyroid cancer. Oncol Res 27: 431-437, 2019.

34. Li YL, Zhao YG, Chen B and Li XF: MicroRNA-132 sensitizes nasopharyngeal carcinoma cells to cisplatin through regulation of forkhead box A1 protein. Pharmazie 71: 715-718, 2016.

35. Sang Q, Yao Z, Wang H, Feng R, Wang H, Zhao X, Xing Q, Jin L, $\mathrm{He} \mathrm{L}, \mathrm{Wu} \mathrm{L}$ and Wang L: Identification of microRNAs in human follicular fluid: characterization of microRNAs that govern steroidogenesis in vitro and are associated with polycystic ovary syndrome in vivo. J Clin Endocrinol Metab 98: 3068-3079, 2013.

36. Kranc W, Budna J, Kahan R, Chachuła A, Bryja A, Ciesiółka S, Borys S, Antosik MP, Bukowska D, Brussow KP, et al: Molecular basis of growth, proliferation, and differentiation of mammalian follicular granulosa cells. J Biol Regul Homeost Agents 31: 1-8, 2017.

37. Thomas FH and Vanderhyden BC: Oocyte-granulosa cell interactions during mouse follicular development: Regulation of kit ligand expression and its role in oocyte growth. Reprod Biol Endocrinol 4: 19, 2006.

This work is licensed under a Creative Commons Attribution-NonCommercial-NoDerivatives 4.0 International (CC BY-NC-ND 4.0) License. 